\title{
EDITORIALS
}

\section{Tackling Care Transitions: Mom and Apple Pie vs. the Devil in the Details}

\author{
Vineet M. Arora, MD, MAPP \\ Section of General Internal Medicine, the Department of Medicine, University of Chicago, Chicago, IL, USA.
}

J Gen Intern Med 24(8):985-7

DOI: $10.1007 / \mathrm{s} 11606-009-1021-\mathrm{x}$

(c) Society of General Internal Medicine 2009

$\mathrm{C}$ ontinuity of care is a fundamental tenet of high quality of care and has been likened to "motherhood and apple pie" ${ }^{1}$. Unfortunately, patients feel that continuity and care transitions are major problems with their healthcare ${ }^{2}$. This is not surprising given the increasing use of specialists, such as hospitalists, which results in increased fragmentation of patient care $^{3}$.Given these trends, investments to improve care transitions are critical to ensuring safe patient care ${ }^{4}$.

For this reason, the Transitions of Care Consensus Policy Statement produced from a collaboration of six medical societies (American College of Physicians, Society of General Internal Medicine, Society of Hospital Medicine, American Geriatrics Society, American College of Emergency Physicians, and Society of Academic Emergency Medicine) is especially timely and welcome $^{5}$. The formal collaboration of these groups is also impressive and unprecedented. This broad involvement of generalists, hospitalists, geriatricians, and emergency medicine physicians increases the likelihood that these recommendations can impact these stakeholder groups. The policy statement represents an important starting point from which to guide improvement initiatives and policy changes to advance care transitions.

However, in considering how to translate these recommendations into practice, there is more work to do. While the consensus statement appropriately recommends a "patientcentered" approach, it is important to note that the patient perspective is missing from this effort. Likewise, perspectives of other healthcare professionals who play an active role in care transitions, including but not limited to case managers, nurses, pharmacists, nursing home staff, and home health and medical transport professionals, are absent. Interestingly, patient advocates and health care providers from these various care settings, have partnered together to form the National Transitions of Care Coalition (NTOCC), which represents 26 groups, to improve care transitions ${ }^{6}$. Future engagement of this broader set of stakeholders is a crucial and necessary step to move these recommendations forward. The policy statement outlines high level-principles for care transitions that are generally agreeable to most physicians, but does not delve into the details of how to improve care transitions. While the recommendations call for actions such as physicians communicating effectively and accessing the medical record during a care transition, little guidance is offered on how to achieve

Published online May 22, 2009 these goals. As with all improvement efforts, the difficulty lies in the implementation of these recommendations or simply, "the devil in the details." At the front lines of patient care, there are numerous practical challenges involved with implementing these recommendations outlined in the consensus statement. There are, however, several additional recommendations outlined below that can help translate this Policy Statement into action for hospitals, office practices, and patients:

\section{EMBRACE ELECTRONIC HEALTH RECORDS AS A TOOL TO IMPROVE CARE TRANSITIONS}

Adoption of an electronic health record has the greatest potential to reform the way in which care transitions are accomplished. National efforts to standardize content for care transitions via electronic health records have already started. The U.S. Department of Health and Human Services Office of the National Coordinator for Health Information Technology, has drafted the "Consultations \& Transfers of Care Draft Detailed Use Case" that includes recommendations for electronic content under different assumptions (perspectives of the receiving physician, transferring physician, patient, etc. ${ }^{7}$. The use cases provide a basis for forming national standards for health information technology solutions to address care transitions.

Unfortunately, these and other national efforts will go to waste if only a select few have the necessary technology to facilitate effective care transitions. The current consensus statement states that improvements in care transitions should not be contingent upon an electronic health record. However, those areas with regional health information organizations (RHIO) and a high adoption of electronic health records can implement these recommendations via interoperable technology solutions, while those organizations and regions without the technology will continue to languish. Given the current investment in electronic health records through American Recovery and Reinvestment Act for meaningful use of electronic health records, the time is now to advocate for adoption of electronic health records functionality and interoperability as a tool to improve care transitions.

\section{REDUCE LOCAL SYSTEM AND CULTURAL BARRIERS TO CARE TRANSITIONS}

Successful adoption of these recommendations depends on local implementation. Specifically, ensuring effective communication during care transitions depends on a culture and a system that support the bidirectional interactive communication endorsed by the policy statement. Even with an electronic health record, technology cannot substitute for the act of verbal communication $^{8}$. Unfortunately, local barriers may impede interactive 
clinician communication during care transitions. At the University of Chicago, we have discovered specific cultural and system barriers that may prevent residents from contacting primary care physicians during a patient's hospitalization. In addition to lack of time, workload, and competing responsibilities, residents report resorting to Google $\mathrm{TM}^{\mathrm{TM}}$ to locate contact information for off-site physicians because it is not available in any system. Residents also report fear that contacting primary care physicians will delay discharge due to additional tests that the primary care physician will request ${ }^{9}$. Implementing broad policy mandates for communication during care transitions without addressing local barriers like these will undoubtedly fail. Such action may even acutely worsen care transitions through promotion of "workarounds," or shortcuts that can bypass barriers that do not address the root of the problem ${ }^{10}$. For example, if it is too difficult or time-consuming to prepare and update the necessary record for a care transition, clinicians may use outdated information that is incorrect during a care transition ${ }^{11}$. In many hospitals, the chart or clinical data repository requires the name of a physician for a patient to be admitted. Unfortunately, this is often the "admitting physician" and may never be updated with the actual physician responsible for a particular patient ${ }^{12}$. A technology solution may actually hasten the use of the incorrect attending instead of the actual physician caring for a patient. This example highlights the need to ensure that information in the medical record during a care transition truly reflects the care process.

Frontline physicians are also looking for tools to use during care transitions ${ }^{13}$. Recently, ensuring effective communication during inter-hospital transfers emerged as a pressing need at the University of Chicago. With the Transitions of Care Policy Statement as a broad guide, our residents needed a point of care tool to obtain the necessary information when accepting transfer patients. Using the mnemonic "PREPARED" (See below), a checklist was introduced to help residents organize the information when receiving a transfer patient. Similar tools for discharge and other types of transitions have already been developed and are being used in the community. Active and faster dissemination of these tools to the medical community is needed so that organizations and practices can learn from experiences of others.

\section{Be "PREPARED" for Transfers}

- Presenting history

- Received therapies (meds, PRBC, nebs, etc)

- Existing baseline (current VS, cognitive status, Hgb, K etc.)

- Pending tests to follow up (cultures, final radiology reads, etc.)

- Anticipated needs $\left(\mathrm{O}_{2}\right.$, access, tele, isolation, consultations)

- Records to be sent (Chart, radiology, EKG, echo, etc.)

- End of life preferences (Code status, POA or surrogate)

- Discussed with family \& PCP? (and their contact info)

\section{IMPLEMENT PATIENT-CENTERED SOLUTIONS FOR PATIENTS MOST AT RISK DURING CARE TRANSITIONS}

For the most vulnerable patients-those who are most at risk during care transitions-the current recommendations do not go far enough. As noted in the Policy Statement, in the absence of major healthcare reform, ensuring appropriate transitional care for patients without a medical home is problematic. While the policy statement recommends that patients be informed about their provider during the care transition, this is very difficult to do in certain patient populations. Among our patient population of mostly low income older African-Americans with limited health literacy, less than $25 \%$ of hospitalized patients were able to correctly identify any inpatient physician in charge of their care ${ }^{14}$.

For these and other groups such as patients with limitedEnglish proficiency, additional solutions to ensure patients understand their illness and their treatment plans during care transitions are needed. Since systems that truly embrace patient centeredness strive to address the needs of different patient populations, patient-friendly tools tailored to the needs of specific groups (cultural, language, etc.) are warranted. Two solutions that have resulted in improved patient outcomes after hospital discharge use coaches to guide patients through their care transition. For example, Dr. Eric Coleman's Care Transitions Intervention employs a transitions coach who visits older patients prior to discharge and then calls patients afterwards ${ }^{15}$. Likewise, investigators at Boston University are using an electronic coach, a friendly avatar named "Louise" to guide patients in their Project RED (Re-engineered discharge) ${ }^{16}$. In contrast to use of coaches for high risk patients, highly educated computer-savvy patients may readily embrace use of electronic personal health records to stay informed of critical information during care transitions. Given the heterogeneity of patient education, illness, resources, and preference, accounting for these differing needs via a "personalized" approach to care transitions is warranted.

\section{DEVELOP AND USE VALID MEASURES OF QUALITY OF CARE TRANSITIONS}

Measuring the quality of care transitions is easier said than done. Most process or outcome measures (readmission, patient awareness, medication adherence, etc) that may initially seem like reasonable measures of quality for a care transition are also subject to multiple patient confounders (patient illness, access to care, adherence, health literacy, etc.) In light of this, it is not surprising that recent data from six academic health centers shows that there was no relationship between patient outcomes and communication with the primary care physician regarding hospitalization ${ }^{17}$. Therefore, using patient reports of their experience during care transitions is a useful adjunct to the macroscopic outcomes. One such measure is the Care Transitions Measure-3, which asks patients about their ability to understand the purpose of their medications and their health needs ${ }^{18}$. These measures of patient experience should be integrated into nationally administered benchmarked patient surveys such as Hospital Consumer Assessment of Healthcare Providers and Systems (HCAHPS) ${ }^{19}$. While the policy statement recommends using such measures for payfor-performance programs, it is important to recognize that subjective reports of the patient experience may be influenced by patient education and health literacy. Therefore, further research is needed before adherence to such measures is linked to reimbursement. 
In summary, the Transitions of Care Consensus Policy Statement represents an important starting point from which to improve care transitions. Now the hard work to implement these guidelines begins. It is only by tackling the "devil in the details" when improving care transitions that we can ultimately enjoy the "mom and apple pie" of improved continuity of care.

Acknowledgements: I would like to acknowledge Julie Johnson, MSPH, PhD from the University of New South Wales, Sydney Australia, Anita Samarth from Clinovations, LLC, and Marshall Chin, MD, MPH, from the Section of General Medicine at the University of Chicago for their helpful insights and contributions.

Corresponding Author: Vineet M. Arora, MD, MAPP; Section of General Internal Medicine, the Department of Medicine, University of Chicago, 5841 S. Maryland Ave. MC 2007, AMB W216, Chicago 60637, IL, USA (e-mail: varora@medicine.bsd.uchicago.edu).

\section{REFERENCES}

1. Kerse N, Mainous A. Continuity through change, a threat or a promise? Journal of the New Zealand Medical Association. 2002;115(1167). Available at: http://www.nzma.org.nz/journal/115-1167/276/. Accessed on March 21, 2009.

2. Leatherman S, McCarthy D. Quality of health care in the United States: a chartbook. The Commonwealth Fund. April 2002; p.99. Available from: http://www.commonwealthfund.org/Content/Publications/Chartbooks / 2002/Apr/Quality-of-Health-Care-in-the-United-States-A-Chartbook. aspx\#citation. Accessed on March 29, 2009.

3. Meltzer D. Hospitalists and the Doctor-Patient Relationship. J Legal Stud. 2001;30(2):589-606.

4. Arora VM, Farnan JM. Care transitions for hospitalized patients. Med Clin North Am. 2008;92(2):315-24.

5. Snow V, Beck D, Budnitz T, et al. Transitions of Care Consensus Policy Statement American College of Physicians-Society of General Internal Medicine-Society of Hospital Medicine-American Geriatrics SocietyAmerican College of Emergency Physicians-Society of Academic Emer- gency Medicine. J Gen Intern Med. 2009; doi:10.1007/s11606-0090969-x.

6. National Transitions of Care Coalition. Available at: http://www.ntocc. org/Home/tabid/36/Home/tabid/36/Default.aspx. Accessed on March 21, 2009.

7. Draft Consultations \& Transfers of Care Draft Detailed Use Case. U.S. Department of Health and Human Services Office of the National Coordinator for Health Information Technology. Available at: http:// www.dhhs.gov/healthit/ahic/materials/02_08/ehr/consultationsTOC. pdf. Accessed on March 21, 2009.

8. Ash JS, Berg M, Coiera E. Some unintended consequences of information technology in health care: the nature of patient care information system-related errors. J Am Med Inform Assoc. 2004;11(2):104-12.

9. Johnson J, Farnan J, Vinci L, Davis A, Arora V. Putting together the missing pieces: A process analysis of PCP-inpatient communication. Midwest Society of General Internal Medicine. 2008

10. Mohr J, Arora V. Break the cycle: Rooting out the workaround. ACGME Bulletin; 2004 Nov. Available at: http://www.acgme.org/acWebsite/ bulletin/bulletin11_04.pdf. Accessed March 29, 2009.

11. Arora V, Kao J, Lovinger D, Seiden SC, Meltzer D. Medication discrepancies in resident sign-outs and their potential to harm. J Gen Intern Med. 2007;22(12):1751-5.

12. Rush JP, Chambers LW, Keddy W. A study to identify who is the responsible physician for each patient in a teaching hospital. QRB Gual Rev Bull. 1986;12(12):426-30.

13. Arora V, Johnson J. A model for building a standardized hand-off protocol. Jt Comm J Qual Patient Saf. 2006;32(11):646-55.

14. Arora V, Gangireddy S, Mehrotra A, Ginde R, Tormey M, Meltzer D. Ability of hospitalized patients to identify their in-hospital physicians. Arch Intern Med. 2009;169(2):199-201.

15. Coleman EA, Parry C, Chalmers S, Min SJ. The care transitions intervention: results of a randomized controlled trial. Arch Intern Med. 2006;166(17): 1822-8.

16. Jack BW, Chetty VK, Anthony D, et al. A reengineered hospital discharge program to decrease rehospitalization: a randomized trial. Ann Intern Med. 2009;150(3):178-87.

17. Bell CM, Schnipper JL, Auerbach AD, et al. Association of communication between hospital-based physicians and primary care providers with patient outcomes. J Gen Intern Med. 2009;24(3):381-6.

18. Parry C, Mahoney E, Chalmers SA, Coleman EA. Assessing the quality of transitional care: further applications of the care transitions measure. Med Care. 2008;46(3):317-22.

19. Jha AK, Orav EJ, Zheng J, Epstein AM. Patients' perception of hospital care in the United States. N Engl J Med. 2008;359(18):1921-31. 